Check for updates

Cite this: RSC Adv., 2017, 7, 18926

Received 24th February 2017

Accepted 21st March 2017

DOI: $10.1039 / c 7 r a 02296 d$

rsc.li/rsc-advances

\title{
Nitrogen doping in the carbon matrix for Li-ion hybrid supercapacitors: state of the art, challenges and future prospective
}

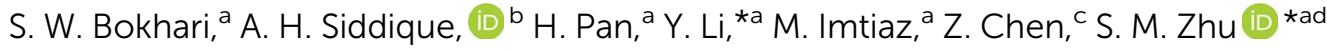
and D. Zhang ${ }^{a}$

Li-ion hybrid supercapacitors (LiHSCs) have emerged as an extremely attractive energy storage system by combining the prime advantages of Li-ion batteries and supercapacitors. As a common electrode material in both lithium ion batteries and supercapacitors, graphene and activated carbons offer a tunable porous structure with high chemical, thermal and physical stability, which in turn results in excellent electronic conductivity and improved capacity as compared with the other electrodes. Elemental nitrogen doping in graphene and activated carbons is believed to further improve their performance. In this review, the state of the art of hybrid supercapacitors is briefly summarized with an emphasis on the use of graphene and activated carbons. Subsequent doping of graphene and activated carbons with nitrogen in LiHSCs is also emphasized.
\end{abstract}

\section{Introduction}

With the increased number of technological devices and the advancements of portable electronics, the global need for smart and efficient energy storage systems is increasing rapidly.

${ }^{a}$ State Key Laboratory of Metal Matrix Complexes, Shanghai Jiao Tong University, 800 Dongchuan Road, Shanghai 200240, P. R. China. E-mail: smzhu@sjtu.edu.cn; Tel: +86-21-34202584

${ }^{b}$ Ningbo Institute of Materials Technology and Engineering (CNITECH), Ningbo, Zhejiang, P. R. China

${ }^{c}$ School of Mechanical, Materials and Mechatronics Engineering, University of Wollongong, Wollongong, NSW 2522, Australia

${ }^{d}$ National Engineering Research Centre for Nanotechnology, Shanghai, P. R. China
Among them, Li ion batteries (LIBs) and supercapacitors are indeed excellent candidates for promising applications in smart energy storage. ${ }^{1,2}$

LIBs are constructed by a Li-ion donating cathode and a Liion accepting anode. ${ }^{3}$ This friendly relationship between the two electrodes facilitates the continuous flow of Li-ions., ${ }^{4,5}$ Hence the capacity and energy of the LIBs is determined by the number of Li-ions accommodated by the electrodes. The most commonly used cathode materials so far are layered Li-metal oxides (LMOs) and the favoured anode is graphite. ${ }^{6}$ The other combinations of electrodes have also been implied. However, the power density of LIBs has not reached a satisfactory level in comparison to the supercapacitor. This is because the power

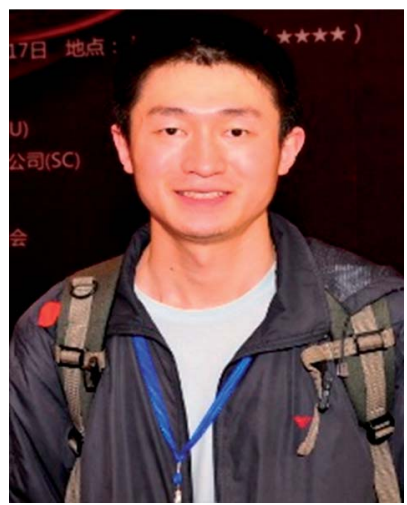

Dr Yao Li was born in 1983 and received his $P h D$ degree in Material Science and Engineering from the Shanghai Jiao Tong University in 2015 under the supervision of Prof. Di Zhang and Prof. Shenmin Zhu. He started working as a research associate in the Shanghai Jiao Tong University in 2015. He is currently working on research of bio-inspired materials and carbon matrix materials, as well as their applications in environmental treatment and energy storage. 
density depends on the nature of electrode reactions i.e. the continuous charge transfer process at the surface of electrodes. The electrodes therefore need to be high in surface area and highly conductive. Another shorting coming of LIBs is that they all have a rather limited cycle life and suffer capacity fading. ${ }^{7}$ The capacity fading is due to the two following reasons: (i) the potential difference between the electrodes that they do not balance each other in the system, which in turn hinders the performance of any of the electrodes. (ii) The deformation of the electrode structure or the deterioration of the electrode from current collector via reaction with electrolyte and the charge carrier. To-date any combination of electrodes used in LIBs can have enhanced the energy density but has a little impact on the power density (power density of LIBs has not reached the high power density of other energy storage devices compared in Ragone plot given in Fig. 1). ${ }^{\mathbf{8 - 1 0}}$

On the other hand, supercapacitors (SCs) have the characteristics of high power density due to their rapid chargedischarge rate and a long life span, which makes them a promising and a very important type of energy storage devices. ${ }^{11-15}$ They can be charged and discharged safely with an ultrahigh cyclic life of around 100000 cycles approximately. A typical supercapacitor is composed of a cathode and an anode separated by an insulating separator in an ion conducting electrolyte. The physical and chemical properties of the electrode materials used in supercapacitors govern their performance. ${ }^{12-16}$

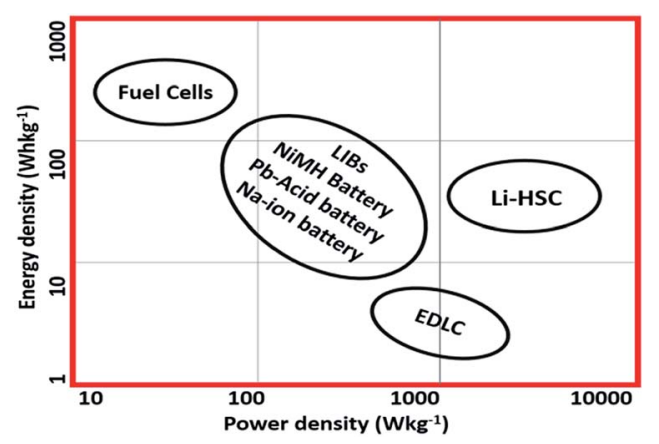

Fig. 1 Ragone plot comparison of different energy storage systems.

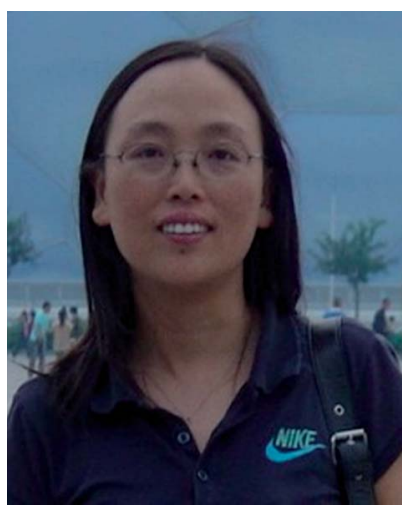

Dr Shenmin Zhu received her $\mathrm{PhD}$ degree from Shanghai Jiao Tong University in 2001. She is presently a professor at the School of Materials Science and Engineering, State Key Lab of Metal Matrix Composites, Shanghai Jiao Tong University. Her current fields of interest are graphene-based functional materials, porous carbon, and bioinspired photonic crystals with stimuli-responsive properties.
Generally, the supercapacitors can be divided into two groups according to the charge storage mechanism; (i) electrochemically double layer capacitors (EDLC) commercially known as supercapacitors and (ii) pseudocapacitors (PC). ${ }^{17-19}$ EDLCs store energy physically by the absorption of electrolyte ions on the surface of active materials. Hence the nature of stored charge is EDLC is electrostatic. Typically an EDLC is composed of two carbon based electrodes separated by a porous membrane and soaked in an electrolyte. On applying a current voltage, cathode and anode attracts solvated anions and cations respectively hence forming an electrochemical double layer where the capacitance on each electrode is given by; ${ }^{20}$

$$
C=\frac{A \varepsilon}{4 \pi d}
$$

where, ' $A$ ' corresponds to the electrochemically active surface area of the electrode material, ' $\varepsilon$ ' denotes the dielectric constant of electrolyte and ' $d$ ' is the thickness of the monolayer of solvent molecules which separates the two layers of the electrochemical double layer. Generally, porous high surface area carbon materials, CNTs etc. are employed as the EDLC electrode materials due to their large surface area and highly porous structure. However, the energy density of EDLC remains a challenge. Currently, commercially available EDLC based on ACs electrode and organic electrolytes provides a cell voltage of up to $2.7 \mathrm{~V}$ with high power density (up to $10^{8} \mathrm{~W} \mathrm{~kg}^{-1}$ ) and good cyclic life but suffer from low energy density due to low capacitance and high ionic resistance of the AC electrode. The research on EDLC electrode materials is now shifted towards novel materials including conducting polymers, nano metal oxides, and heteroatom doped carbon materials. ${ }^{21-24}$

Pseudocapacitors (PCs), on the other hand, store energy via faradic charge storage mechanism occurring due to the reversible reactions between the electrolyte and electrode interfaces. Typically, electrodes materials of PCs are composed of Transition Metal Oxides (TMOs) and conducting polymers. The reported capacitance of a typical PC based on TMO electrode is $300-1000 \mathrm{~F} \mathrm{~g}^{-1}$ but their cyclic performance is very unstable hence the capacity fading is obvious over a few number of cycles. ${ }^{25,26}$

From the analysis above, it is known that both LIBs and supercapacitors suffer from challenges that limit their performance. ${ }^{27} \mathrm{Li}$ ion batteries offer a higher energy density of about $>200 \mathrm{~W} \mathrm{~h} \mathrm{~kg}^{-1}$ but their poor life cycle and low power density $<10^{3} \mathrm{~W} \mathrm{~kg}^{-1}$ limit their performance to meet the ever-increasing demand. ${ }^{28}$ On the other hand, supercapacitors, though provide a very high power density i.e. $>10^{3} \mathrm{~W} \mathrm{~kg}^{-1}$ and a long cyclic stability but their energy density is very lower i.e. $5-10 \mathrm{~W} \mathrm{~h} \mathrm{~kg}^{-1}$ only. ${ }^{29}$

The possible solutions to the problems with the two energy storage systems i.e. LIBs and SCs are (i) either to research new electrode materials with enhanced electrochemical performance ${ }^{30,31}$ and life cycle ${ }^{32,33}$ or (ii) to combine both systems into a single device ${ }^{34-36}$ resulting in a hybrid system which could possess the advantageous properties of both LIBs and SCs, overcoming the short comings that limit the performance of both systems. ${ }^{37-40}$ 
Li-ion capacitors (LICs), also known as hybrid supercapacitors (HSCs), are designed to bridge the gap between the traditional LIBs and SCs and to combine the prime advantages of high energy density of LIBs and high power density and cyclic stability of SCs in a single device. ${ }^{31}$ The comparison of Li-ion hybrid supercapacitors (LIHSC) with the other energy storage systems is shown in Fig. 1 which depicts that the hybrid supercapacitors are indeed superior to the other energy storage systems owing to their enhanced energy density coupled with higher power density. ${ }^{\mathbf{4 1 , 4 2}}$

\section{Li-ion hybrid supercapacitor}

\subsection{The mechanism of the hybrid supercapacitor}

Li-ion hybrid supercapacitor is constructed by incorporating a battery type electrode (anode) and a capacitor type electrode (cathode) in an electrolyte containing $\mathrm{Li}$ ions $\left(\mathrm{Li}^{+}\right) \cdot{ }^{40} \mathrm{Li}^{+}$ions from the electrolyte intercalate through a very high surface area cathode material. While on the surface of the anode, ions from the electrolyte accumulate hence creating a pseudo-capacitance mechanism. Both electrodes function reversibly in different potential windows hence resulting in an improved performance. The unique characteristics of both the electrodes result in a different and an improved charge storage mechanism. ${ }^{39}$

Fig. 2 explains the working mechanism of the hybrid device. During charging, $\mathrm{Li}^{+}$from the electrolyte accumulates on the surface of the negative electrode (anode) and the negative ions moves towards the positive electrode (cathode). An EDLC mechanism is also observed by the accumulation of charges on the surfaces of cathode. Hence, cathode acts an EDLC electrode and anode functions as a PC electrode. The reverse phenomenon is observed during discharging. LIHSCs though inculcate the advantageous feature of both LIBs and SCs, yet they differ from both components in a distinguishable way. In comparison with the traditional LIBs, non-aqueous electrolyte is mostly used in the HSCs which widens the working potential window of the hybrid device and a larger capacity is provided by the LIB type electrode. Hence a superiorly higher energy density is

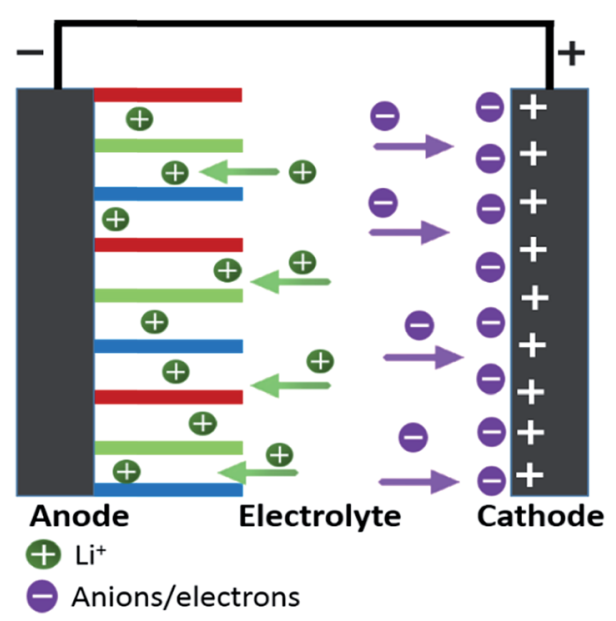

Fig. 2 Mechanism of working of LIHSC. achieved. ${ }^{42}$ Whereas, in comparison with the SCs, the electrochemical double layer electrode advances the power capability and cyclic stability. ${ }^{\mathbf{4 3}}$

In principle, a capacitor-type electrode (cathode) and a lithium ion battery-type electrode (anode) in a $\mathrm{Li}^{+}$containing electrolyte is the simplest assembly of a LiHSC. ${ }^{44}$ In LIHSC, adsorption and desorption of ions occurs on cathode and the intercalation of $\mathrm{Li}^{+}$occurs on anode. Both electrodes work simultaneously in an appropriate operational voltage and the device attains a better energy density at a higher power density and retains its performance over a large number of electrochemical cycles. ${ }^{45}$

LIHSCs can be operated in both aqueous and non-aqueous electrolytes. Non-aqueous system is of great importance because of charging-discharging at a fast rate and higher cyclic stability. ${ }^{46-48}$ The combination of two distinct energy storage mechanisms i.e. faradic and non-faradic in a single device is what makes LIHSCs a superior energy storage system with unique characteristics and enhanced performance..$^{\mathbf{4 4 9}}$

The "generation-II supercapacitors"-the first ever commercialized Li-ion hybrid supercapacitor which have been very famous in early 2000, were constructed by using graphite as a cathode and activated carbon as an anode material. The cell voltage used was $3.8 \mathrm{~V}$, and the system exhibited $20 \mathrm{~W} \mathrm{~h} \mathrm{~kg}$ specific energy, and $3000 \mathrm{~W} \mathrm{~kg}^{-1}$ power density. The average electrode capacitance was $4.2 \mathrm{~F} \mathrm{~cm}^{-3}$ (three times better than the conventional EDLC i.e. $1 \mathrm{~F} \mathrm{~cm}^{-3}$ ) retaining $90 \%$ capacity over 1500 cycles even at elevated temperatures. So it has a 3 fold better performance than LIBs and SCs i.e. 3 fold better energy density than supercapacitors and 3 fold better power density than Li-ion batteries. ${ }^{50}$

\subsection{The composition of the hybrid supercapacitors}

As is mentioned above, LiHSCs are composed of a cathode and

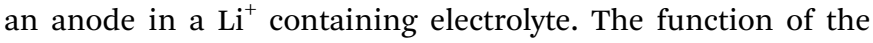
capacitor type electrode is to adsorb/de-adsorb the ions, and that of the battery type electrode is to insert/de-insert Li ions. Both electrodes perform reversible mechanisms in different potential ranges, hence resulting in a fast charge rate, enhanced cyclic life, and a high energy density. In general, a hybrid capacitor should have higher energy density than a battery. That means the use of new types of capacitor electrode could greatly enhance the energy density of LIHSC.

The following equation can be used to determine energy and power densities of the hybrid systems; ${ }^{51-53}$

$$
\begin{gathered}
E=\frac{1}{2} C V^{2} ; \\
P=\frac{V^{2}}{4 R}
\end{gathered}
$$

From above equations, it is clear that the operating voltage ' $V$ ' is of great significance because energy density ' $E$ ' \& power density ' $P$ ' both are proportional to $V^{2}$. Hence, optimization of the operating voltage is a crucial factor to enhance the 
electrochemical performance of the hybrid system. ${ }^{54-57}$ The electrolytes used in Li-ion capacitors consist of lithium salts in organic solvents allowing for a large operating voltage..$^{58}$ It has been reported that a high cyclic stability is achieved by using a narrower voltage window at the cost of energy density. A higher voltage range can be achieved in LiHSC because two distinct electrochemical mechanisms are governed by the reversible operations of the two electrodes functioning under different potential ranges. Commercial EDLCs steer at $2.7 \mathrm{~V}$ in organic electrolyte system and exceeding this voltage window leads to side reactions consequentially harming the EDLC cell. Contrary, LiHSC can achieve a voltage window of over $4.0 \mathrm{~V}$, hence the energy density is enhanced three fold over the EDLC. Organic electrolytes (2.5-3.0 V) and aqueous/non-aqueous ionic electrolytes $(4.0 \mathrm{~V})$ are more desirable than aqueous electrolyte because they allow for an elevated voltage window. The specific capacitance ' $C$ '; and electron spin resonance (ESR) ' $E$ ' are also crucial factors for improving the energy and power density of LiHSC. ' $C$ ' directly correlates to the pore size distribution (PSD) and specific surface area (SSA) whereas the ESR is regulated by the conductivity of the electrode material and electrolyte, and contact resistance between current collector and electrode material. Hence, these factors need to be considered for designing an appropriate high energy and power density LiHSC. ${ }^{77,58}$

The battery type electrode materials that have been used previously include some carbonaceous materials, metal oxides (MOx) and metal hydroxide (MHOx) etc. And the capacitor type electrode materials commonly used in LIHSC are constructed from the activated carbons (ACs) due to their manageable porous structure and a high surface area. Other materials like carbon nanotubes (CNTs) and graphene are also used. ${ }^{59}$

Graphene and activated carbons have been widely investigated as the electrode materials for the Li-ion hybrid supercapacitors owing to their high surface area and great vulnerability to the $\mathrm{Li}$ ion intercalation, superior capacity retention, long life cycle and the confined structure conformation. ${ }^{60,61}$ The hybrid devices assembled using electrodes based on graphene or activated carbon and their composites have reported to exhibit a high energy density coupled with a high power density. A long stable cyclic life and improved capacity retention has also been reported. ${ }^{62,63}$

To improve the properties of graphene and activated carbons based electrodes, morphology control and element doping have been used. 0-D graphene quantum dots, ${ }^{64-66}$ 1-D graphene nanoribbons (GNRs), ${ }^{67}$ 2-D graphene sheets, 3-D network of graphene ${ }^{68,69}$ and porous graphene ${ }^{70,71}$ have been prepared. 3D crosslinked structure of graphene has been introduced to achieve an improved performance, where the crosslinking sites are created by $\pi-\pi$ stacking of graphene sheets. Recently, Cheng et $a .^{72}$ reported 3D printed graphene aerogels with $90 \%$ compressibility, ultra-light weight and high conductivity (Fig. 3). This kind of method can be used to print the desired structural configuration of graphene network to smoothen the path of electron and Li-ions in a hybrid capacitor. As for the elemental doping method, such as N-, Cr, B-doping in graphene

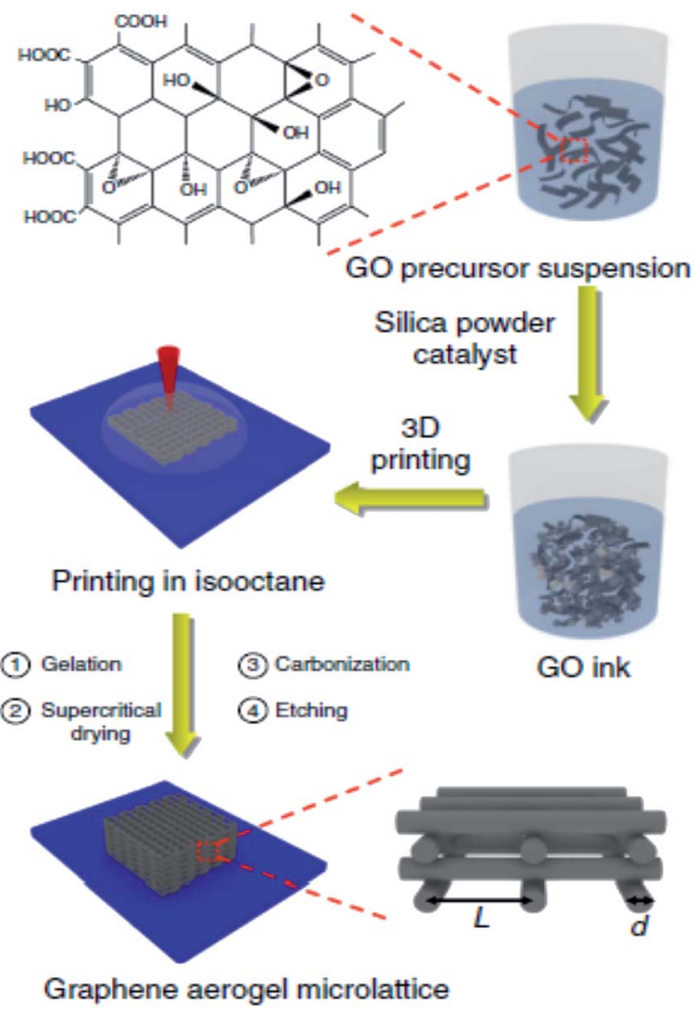

Fig. 3 Schematic of the fabrication process. Desirable GO ink was prepared by mixing GO suspension with fumed silica powder and $\left(\mathrm{NH}_{4}\right)_{2} \mathrm{CO}_{3}$ or $\mathrm{R}-\mathrm{F}$ catalyst solution. A micro-nozzle, immersed in isooctane solution to prevent drying, was used to extrude the GO ink. The excess liquid was evaporated by super-critically drying the as printed micro lattice. The printed structure was carbonized at $1050^{\circ} \mathrm{C}$ under $\mathrm{N}_{2}$. Finally, HF was used to etch the silica filler. Reproduced with permission $^{72}$ (c) 2016 The Nature Communication.

and activated carbon has been reported with improved electrochemical performance. ${ }^{73-75}$

This review focuses on the state-of-art of nitrogen doped graphene and activated carbon electrodes so far applied in hybrid supercapacitors. The effect of nitrogen doping on the enhanced electrochemical activity of the flexible electrodes is reported. And an outlook of the possible future direction is also presented.

2.2.1 N-doped materials. Nitrogen is a pseudo capacitive element. Nitrogen doping is considered to be an ideal method of inculcating pseudo-capacitive property into graphene and activated carbons for applications in high performance supercapacitors. ${ }^{76}$ The electronegativity difference between nitrogen and carbon is relatively high i.e. $3.04: 2.55$, which results in the polarization of carbon network in $\mathrm{N}$-doped graphene (NG) sheets. This polarization induces an "activation region", by influencing the spin density and charge distribution of the carbon atoms, which directly catalyse the electrochemical reactions taking place on the surface of NG. The band gap between the conduction band and valence band is opened by the nitrogen dopant which shifts the Fermi level above the Dirac point. This band gap makes NG a suited candidate for electronic and semiconducting applications. Briefly, as a result of 
the polarization, electronic, magnetic, optical, electrical and electrochemical properties of graphene are altered. The possible bonding configurations of NG are shown in Fig. $4 .^{77}$

There are two chief ways of preparing NG networks; in situ doping or direct synthesis and ex situ doping. In situ doping or direct synthesis is the simultaneous doping and structuring of the carbon network by adding the nitrogen precursor during the solvothermal and CVD growth of graphene. ${ }^{78,79}$ In ex situ doping or in other words post treatment, graphene or graphene oxide is treated with nitrogen precursor by annealing or plasma treatment to get NG. ${ }^{80}$ Ammonia $\left(\mathrm{NH}_{3}\right)$. Melamine $\left(\mathrm{C}_{3} \mathrm{~N}_{6} \mathrm{H}_{6}\right)$, urea $\left(\mathrm{CH}_{4} \mathrm{~N}_{2} \mathrm{O}\right)$, dicyandiamide $\left(\mathrm{C}_{2} \mathrm{H}_{4} \mathrm{~N}_{4}\right)$ and graphitic carbon nitride $\left(\mathrm{g}-\mathrm{C}_{3} \mathrm{~N}_{4}\right)$ are the mostly used nitrogen precursors. ${ }^{\mathbf{8 1 , 8 2}}$ Nitrogen usually forms three bonding configurations in graphene lattice i.e., pyridinic $(398.5 \mathrm{eV})$, pyrrolic $(400.0 \mathrm{eV})$ and graphitic nitrogen $(401.2 \mathrm{eV})$ which shift according to the doping precursors and annealing conditions. ${ }^{\mathbf{8 3 - 8 5}}$

Electron rich $\mathrm{N}$-atoms in NG improve the electronic conductivity and create holes in the graphene sheets. This improves the electrolyte wetting and speeds up the electrolyte ion migration forming a double layer on the surface of electrode. ${ }^{\mathbf{8 6 , 8 7}}$ Also, nitrogen provides additional active sites for lithiation by inducing defects thereby enhancing the capacitive efficiency of NG electrodes. ${ }^{\mathbf{8 8}, \mathbf{8 9}}$

Nitrogen doping in activated carbon results in an increased specific capacitance. ${ }^{90}$ The improved wettability of pores due to faradic reaction of nitrogen containing functional groups not only increases the specific capacitance but also increase the conductivity of the nitrogen doped activated carbons. To dope nitrogen, ammonia $\left(\mathrm{NH}_{3}\right)$ is most commonly used during heat treatment of the activated carbon.

Nitrogen doping in graphene and in activated carbon further enhances the electrochemical activity of the electrodes assembled using NG or nitrogen doped activated carbon (NAC) and their composites. The hybrid device based on NG and NAC electrode has no doubt possessed an increased electrochemical performance. ${ }^{\mathbf{9 1 , 9 2}}$

\subsection{Graphene based electrodes in the hybrid supercapacitor}

Graphene has occupied most of the research in the field of energy storage due to its exceptionally superior properties. There are an abundant number of articles published every other day revealing a new aspect of graphene and graphene based composites in energy storage and conversion devices. ${ }^{93,94}$

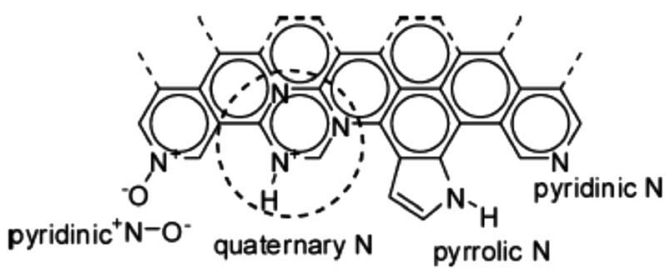

Fig. 4 Bonding configurations of $\mathrm{N}$-atoms in NG. Reproduced with permission ${ }^{77}$ @ 2012 American Chemical Society.
The thin monolayer graphene offers a useful platform for developing highly capacitive hybrid energy storage devices, with experimental specific surface area of $1500 \mathrm{~m}^{2} \mathrm{~g}^{-1}$, high electrical conductivity of $106 \mathrm{~S} \mathrm{~cm}^{-1}$, as well as inherent flexibility and tuneable structure. Notably, the $744 \mathrm{~mA} \mathrm{~h} \mathrm{~g}^{-1}$ theoretical specific capacity, $550 \mathrm{~F} \mathrm{~g}^{-1}$ specific capacitance and an ultrahigh surface-to-volume ratio make graphene an ideal material for faster transportation of ionic species and electric charges in LIBs and SCs and consequently the LIHSC. ${ }^{\mathbf{9 5 , 9 6}}$

In EDLCs, the electrode materials should be stable i.e. should not undergo any faradic reactions and specially should have high Specific Surface Area. ${ }^{97}$ In pseudocapacitors, the electrode materials should be rich in pseudocapacitive elements. GO has a large number of oxygen groups which makes it suitable for pseudo-capacitor applications. ${ }^{98}$ Also, the perfect tuneable pore structure of graphene facilitates the movement of Li-ion and electrolyte ions in LIBs and consequently the performance is improved.99-102

LIHSC based on graphene materials are reported to achieve superior performance: a high energy density and power density as well as rapid charging and discharging over a large number of cycles. ${ }^{103-107}$

Zhang et al. ${ }^{108}$ assembled a Li-ion capacitor using a 3D graphene based porous carbon material as an anode and flash reduced graphene oxide as a cathode material exhibiting an excellent performance with a high operating voltage of $4.2 \mathrm{~V}$, high energy density of $148.3 \mathrm{~W} \mathrm{~h} \mathrm{~kg}^{-1}$ at a power density of $141 \mathrm{~W} \mathrm{~kg}^{-1}$ with a maximum power density of $7800 \mathrm{~W} \mathrm{~kg}^{-1}$ obtained at an energy density of $71.5 \mathrm{~W} \mathrm{~h} \mathrm{~kg}^{-1}$. This excellent performance is attributed to the all graphene based electrode system taking full advantage of graphene. A one pot sol-gel method was reported for the fabrication of graphene-lithium titanate composite by dispersing graphite oxide in aqueous solution of lithium acetate and tetra butyl titanate and annealing in $\mathrm{H}_{2} / \mathrm{Ar}^{109}$ The LIHSC assembled by using it as a cathode with activated carbon as an anode delivered a specific
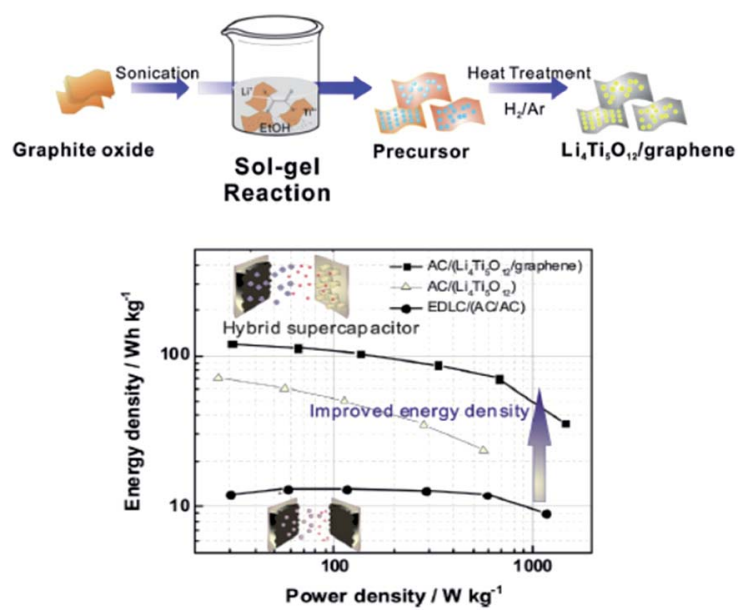

Fig. 5 Schematic diagram of the synthesis of $\mathrm{Li}_{4} \mathrm{Ti}_{5} \mathrm{O}_{12} /$ graphene composites and the electrochemical performance of the hybrid electrochemical supercapacitor. Reproduced with permission ${ }^{109}$ (c) 2015 Elsevier 
capacity of $126 \mathrm{~mA} \mathrm{~h} \mathrm{~g}^{-1}$ at $20{ }^{\circ} \mathrm{C}$ and a high energy density of $120.8 \mathrm{~W} \mathrm{~h} \mathrm{~kg}^{-1}$ when the power density reached $1500 \mathrm{~W} \mathrm{~kg}^{-1}$ (Fig. 5). However, a binder used during the electrode formation lessens the overall capacity of the electrode materials. Therefore, a binder free electrode has also been reported based on graphene nanosheet (GNS) coated 3D nickel foam (NF) anchored by hierarchical flower-like NiAl layered double hydroxide (LDH). The as- constructed LIHSC device using LDHNF/GNS and NF/GNS as anode and cathode delivered $31.5 \mathrm{~W} \mathrm{~h}$ $\mathrm{kg}^{-1}$ energy density at the power density of $400 \mathrm{~W} \mathrm{~kg}^{-1}$ and a specific capacity of $67.2 \mathrm{C} \mathrm{g}^{-1}$ at $5 \mathrm{~A} \mathrm{~g}^{-1}$ after 5000 cycles with $80 \%$ retention.

Reduction agents can have a greater impact on the performance of graphene based materials as they play a great role in surface tuning of graphene oxide. Lee, Ji Hoon, et al. ${ }^{\mathbf{1 1 0}}$ used urea reduced functionalized graphene oxide to improve the capacities of activated carbon cathodes which hinders the performance of conventional graphite/AC LIC. Their LIC delivered a specific energy density of approximately $106 \mathrm{~W} \mathrm{~h} \mathrm{~kg}$ total $^{-1}$ and a specific power density of approximately $4.2 \mathrm{~kW} \mathrm{\textrm {kg } _ { \text { total } }}{ }^{-1}$ with cyclic stability up to 1000 cycles. This kind of performance can be attributed to the formation of amide functional groups during urea reduction process which improved the specific capacity $37 \%$. This kind of surface modification facilitated the enolization process for the lithiation/delithiation. This method opens up new horizons for the appropriate functionalization of graphene/graphene oxide and their role in the LiHSC industry. ${ }^{\mathbf{1 1 1}}$

2.3.1 Electrodes based on nitrogen doped graphene. Elemental nitrogen doping has been employed to improve the conductivity and induce the pseudo capacitance. The first use of nitrogen doped graphene as an anode material for LIHSC was reported by Ragupathy group. ${ }^{112}$ The specific capacitance of their asymmetric hybrid device was measured to be $72 \mathrm{~F} \mathrm{~g}^{-1}$ at a current density of $0.5 \mathrm{~mA} \mathrm{~cm}{ }^{-2}$ with octahedral shaped high voltage material $\mathrm{LiNi}_{0.5} \mathrm{Mn}_{1.5} \mathrm{O}_{4}$ as the cathode material. The device was reported to provide an energy density of $15 \mathrm{~W} \mathrm{~h} \mathrm{~kg} \mathrm{~kg}^{-1}$ and a power density of $110 \mathrm{~W} \mathrm{~kg}^{-1}$. They have reported that a HSC based on a composite cathode and an NDG anode can be tuned to an expanded voltage of up to $1.3 \mathrm{~V}$. The uniqueness of their hybrid device was the remarkable capacity retention of $100 \%$ after 2300 cycles. However, for commercial applications, current leakage and self-discharge are the vital factors which need to be considered. Fig. 6 shows the schematic illustration of their device and its performance evaluation.

Nitrogen doped graphene can be used not only as a negative electrode material but also a positive electrode material in LiHSC with excellent performance and long stable cycle life.

Zhang group ${ }^{\mathbf{1 1 3}}$ has used nitrogen doped graphene as a cathode material and Li-ion intercalated compound $\mathrm{LiMn}_{2} \mathrm{O}_{4}$ as an anode material in $2 \mathrm{M} \mathrm{LiNO}_{3}$ electrolyte in a LiHSC which exhibited an energy density of $22.15 \mathrm{~W} \mathrm{~h} \mathrm{~kg}^{-1}$ and a high power density of $2000 \mathrm{~W} \mathrm{~kg}^{-1}$. The capacity retention was $80 \%$ after 2000 galvanostatic cycles. However, the energy density at a higher power density needs to be improved before considering this system for practical application (Fig. 7).

\subsection{Electrodes based on nitrogen doped activated carbons}

Activated carbon is a highly porous material and therefore it facilitates the movement of ions through the pores. ${ }^{113}$ Activated carbons can be defined as the carbon materials abundant in any/or all three types of pores i.e. micropores, mesopores and macropores. The environmental friendliness, high SSA, tailorable morphology, and high electrical conductivity of activated carbon have resulted in its tremendous successes as an electrode material in energy storage devices. ${ }^{\mathbf{1 1 4}}$

The electrochemical performance of activated carbon rely on the carbon precursor and the activation methodologies. ${ }^{\mathbf{1 1 3 , 1 1 4}}$ Moreover, pore size, pore volume and the amount of electrochemically activating agents (EAAs) in activated carbon can also be controlled and changed depending on the reaction protocols which in turn alters its electrical conductivity and electrical capacity. ${ }^{\mathbf{1 1 5}}$ Notable capacitive performance have been achieved by activated carbons derived from biomass e.g. waste tea and coffee, corn, rise husk, sugar cane bagasse, banana fibres and pinecones etc. ${ }^{\mathbf{1 1 6 , 1 1 7}}$ Nevertheless, the activity of the ACs is restricted by their low electrical conductivity and large number of micro pores preventing the migration of the electrolyte. Carefully modulating the structure of the ACs can tackle this problem and result in an even higher capacitance than reported to date. ${ }^{118,119}$

Many authors have investigated the properties of HSC using activated carbons and their derivatives as electrodes. Bakhmatyuk, B. P. ${ }^{\mathbf{1 2 0}}$ investigated commercial activated carbon material as a polarized electrode of LIC in ionic $\mathrm{ZnCl}_{2}$ electrolyte. They reported an experimental pseudocapacitive discharge capacity of $7673 \mathrm{~F} \mathrm{~g}^{-1}$ with a cyclic efficiency of $93 \%$. When tested practically, the device had a specific capacity of $1254 \mathrm{C} \mathrm{g}^{-1}$, specific energy $396 \mathrm{~W} \mathrm{~h} \mathrm{~kg}^{-1}$ and specific power $2280 \mathrm{~W} \mathrm{~kg}^{-1}$, which $82-87 \%$ is aligning with the theoretical value. Babu et $a .^{\mathbf{1 2 1}}$ fabricated porous carbon from rice husk and used two acids $\mathrm{KOH}$ and $\mathrm{H}_{3} \mathrm{PO}_{4}$ to activate it separately. They compared both activated carbons by using as a cathode against an insertion type $\mathrm{Li}_{4} \mathrm{Ti}_{5} \mathrm{O}_{12}$ (LTO) as an anode. They achieved a maximum energy density of $\sim 57 \mathrm{~W} \mathrm{~h} \mathrm{~kg}^{-1}$ and $37 \mathrm{~W} \mathrm{~h} \mathrm{~kg}^{-1}$ by $\mathrm{KOH}$ and phosphoric acid activated LIHSC respectively. The $\mathrm{KOH}$ activated carbon based LIHSC maintained an energy density of $45 \mathrm{~W} \mathrm{~h} \mathrm{~kg}^{-1}$ at a higher power density of $4300 \mathrm{~W} \mathrm{~kg}^{-1}$. The capacity retention was reported to be $92 \%$ after 2000 cycles at a high current density of $2 \mathrm{~A} \mathrm{~g}^{-1}$. This makes rice husk based porous carbon a good candidate to be considered as the cathode materials for LIHSC.

Lemon peel has also been used for the preparation of porous carbon. ${ }^{122}$ When used as an EDLC type negative electrode with a novel $\mathrm{NiCo}_{2} \mathrm{O}_{4}$ nanograss (NG)-array coated carbon fibre $\left(\mathrm{NiCo}_{2} \mathrm{O}_{4} \mathrm{NG@CF}\right)$ as a pseudocapacitive positive electrode. The resultant LIHSC got a maximum specificity capacity of $17.5 \mathrm{~F}$ $\mathrm{g}^{-1}$ and an energy density of $6.61 \mathrm{~W} \mathrm{~h} \mathrm{~kg}^{-1}$ at the current of 1 $\mathrm{mA}$ and a power density of $425 \mathrm{~W} \mathrm{~kg}^{-1}$ with a capacity retention of $92 \%$ over 3000 cycles. The power density of the device was very low. However, an LED was successfully lit up by connecting three fibre HSC in series even in folded conditions. So this scheme can be good for wearable electronic applications if the 

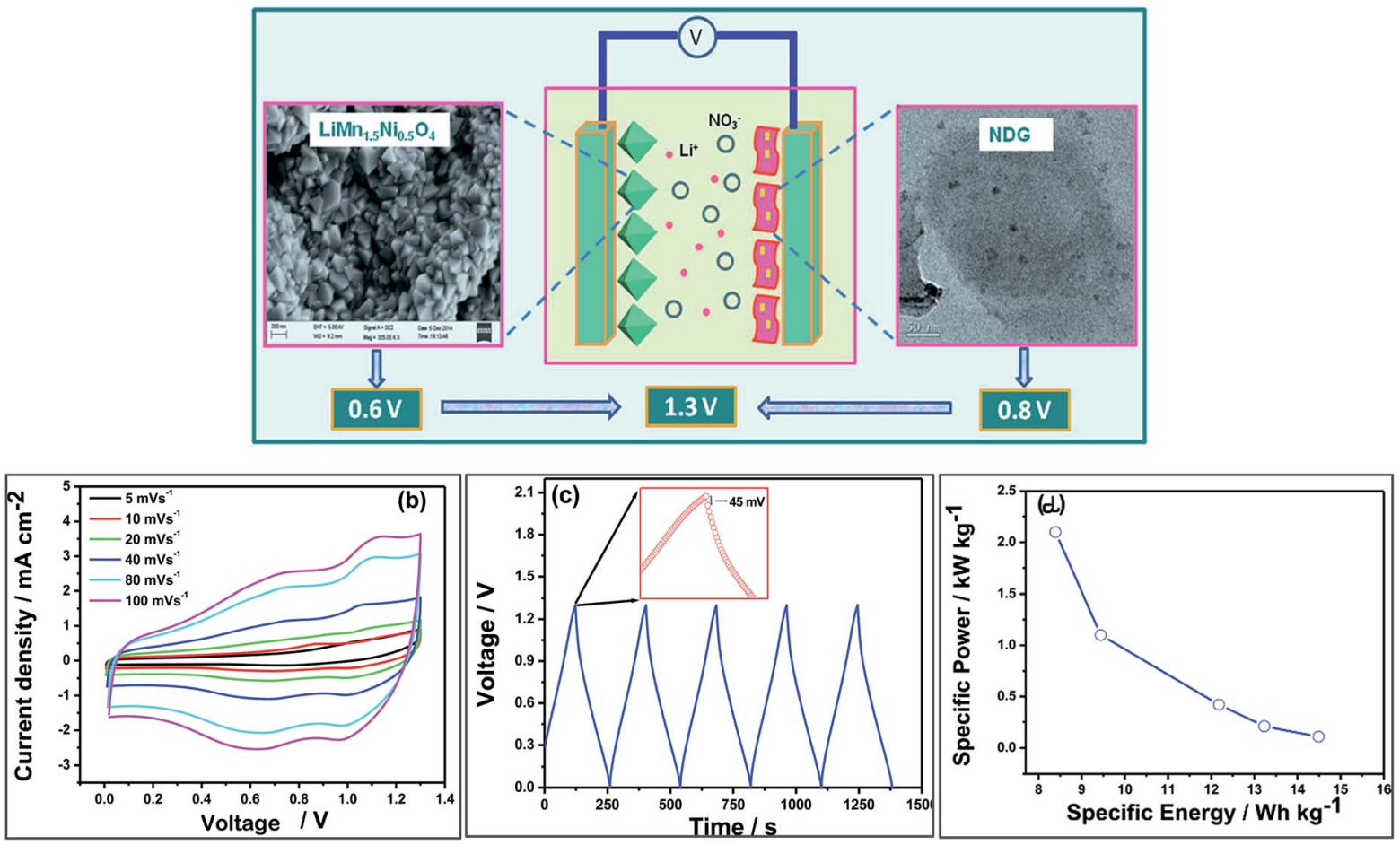

Fig. 6 (a) LiHSC consisting of nitrogen doped graphene as an anode at $\mathrm{LiNi}_{0.5} \mathrm{Mn}_{1.5} \mathrm{O}_{4}$ as a cathode material. (b) CVs of the asymmetric device assembled using nitrogen doped graphene as an anode material and LiNi ${ }_{0.5} \mathrm{Mn}_{1.5} \mathrm{O}_{4}$ as a cathode material in $3 \mathrm{M} \mathrm{LiNO}_{3}$ electrolyte. (c) Galvanostatic charge-discharge curves recorded at $0.5 \mathrm{~mA} \mathrm{~cm}^{-2}$. (d) Ragone plot of the hybrid cell. Reproduced with permission. ${ }^{112} \odot 2015$ Royal Society of Chemistry.

power density is improved. A hybrid supercapacitor using activated carbon as cathode and urchin like titania $\left(\mathrm{TiO}_{2}\right)$ as an anode material attained an energy density of $50.6 \mathrm{~W} \mathrm{~h} \mathrm{~kg}^{-1}$ at a power density of $194.4 \mathrm{~W} \mathrm{~kg}^{-1}$ with a reaction time of $20 \mathrm{~h}$. The capacity was reported to be $62.5 \mathrm{~F} \mathrm{~g}^{-1}$ and an $89 \%$ capacity retention after 5000 cycles at $3 \mathrm{~A} \mathrm{~g}^{-1}$. It is anticipated that this asymmetric setup can find applications in electrical vehicles.

Makino et al. ${ }^{123}$ assembled LiC incorporating water-stable multi-layered lithium-doped carbon $\left(\mathrm{Li}_{x} \mathrm{C}_{6}\right)$ cathode and an activated carbon based anode. They used poly(ethylene oxide)lithium-bis(trifluoromethansulfonyl) imide (LiTFSI) polymer electrolyte containing $N$-methyl- $N$-propylpiperidinium- bis(trifluoromethansulfonyl) imide (PP13TFSI) ionic liquid and have achieved a specific capacity of $20.6 \mathrm{~mA} \mathrm{~h} \mathrm{~g}^{-1}$ within a voltage window of $2.7-3.7 \mathrm{~V}$, and a capacity retention up to 3000 cycles. They also designed an aqueous LiHSC using $\mathrm{RuO}_{2}$ nanosheet anode and multi-layered $\mathrm{Li}_{x} \mathrm{C}_{6}$ cathode with PEOLiTFSI PP13TFSI. This setup showed specific capacity of 196

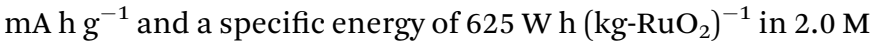
acetic acid-lithium acetate buffered solution at $25{ }^{\circ} \mathrm{C}$. The maximum specific capacity was reported to be $900 \mathrm{~F} \mathrm{~g}^{-1}$. This comparative study demonstrated the advantages of pre-lithium doped electrodes which may result in higher specific capacity
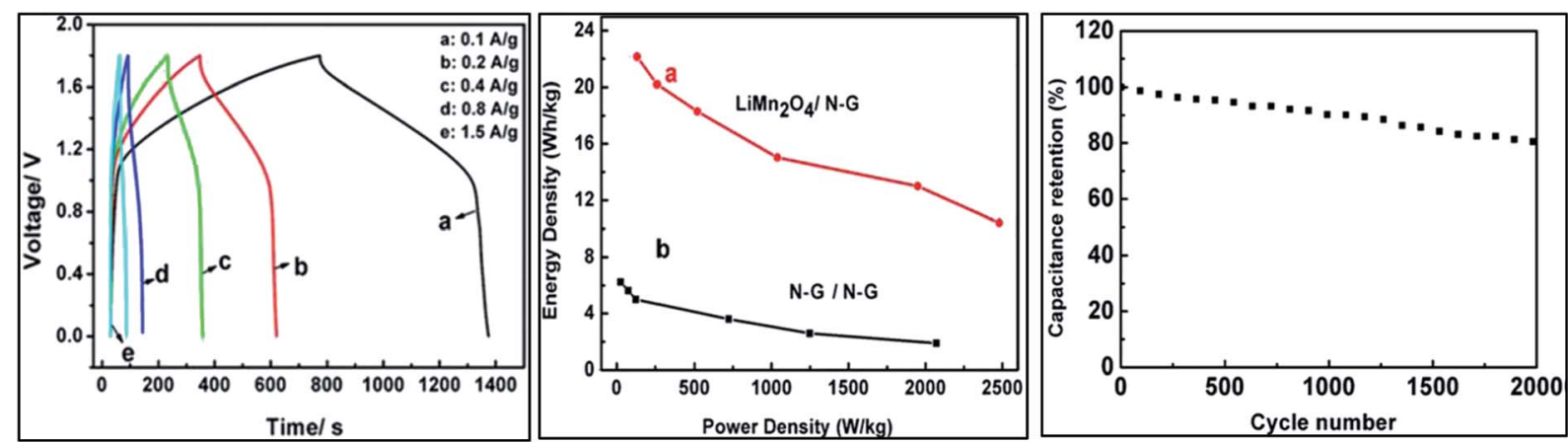

Fig. 7 (a) Galvanostatic charge-discharge curves of LiHSC consisting of NG as a cathode and $\mathrm{LiMn}_{2} \mathrm{O}_{4}$ as an anode. (b) Ragone plot (c) cycling stability of LiHSC at a current rate of $2 \mathrm{~A} \mathrm{~g}^{-1}$ between $0-1.8 \mathrm{~V}$. Reproduced with permission. ${ }^{113}$ (c) 2015 The Electrochemical Society. 
due to the smooth insertion of Li-ions. The assembly of the hybrid device is shown in Fig. 8.

Lim et al. ${ }^{124}$ synthesized $\mathrm{Nb}_{2} \mathrm{O}_{5}$ @carbon core-shell nanocrystals $\left(\mathrm{Nb}_{2} \mathrm{O}_{5} @ \mathrm{C} \mathrm{NCs}\right)$ in a one pot water using oil micro-emulsion system and used them as high-power anode materials for LIC. Manipulating the parameters of the micro emulsion, a desired structure can be obtained. The reversible specific capacity achieved by using this anode is reported to be $180 \mathrm{~mA} \mathrm{~h} \mathrm{~g}^{-1}$ at $0.05 \mathrm{~A} \mathrm{~g}^{-1}$ in 1.1 to $3.0 \mathrm{~V}$ against $\mathrm{Li} / \mathrm{Li}^{+}$.

The above examples show that the topology of the activated carbons plays a vital role in the output of the HSC devices. The narrow pore size distribution and controlled pore structure as well as high SSA govern better performance of the HSC because the large surface areas will result in a large specific capacitance, and the narrow pore size distribution and controlled pore structure facilitate the electrolyte and ion movement through the pores during the charge/discharge process. ${ }^{\mathbf{1 2 5}}$

The performance of activated carbon can be further improved by the $\mathrm{N}$ doping of the activated carbon. Zhang group $^{36}$ has reported the use of nitrogen doped activated carbon (a very high surface area up to $2900 \mathrm{~m}^{2} \mathrm{~g}^{-1}$ with $4 \mathrm{wt} \%$ nitrogen) for the first time as a cathode material for a LiHSC where $\mathrm{Si} / \mathrm{C}$ was used as an anode material in an organic electrolyte under a cathode to anode mass ratio of $2: 1$. They prepared nitrogen doped activated carbon via one step process using ammonia as nitrogen precursor and pre-treated corncobs as precursor for the activated carbon and annealed at different temperatures. They achieved energy density of 230-141 W h $\mathrm{kg}^{-1}$ at $1747-30127 \mathrm{~W} \mathrm{~kg}^{-1}$ power density. The LiHSC achieved $76.3 \%$ cyclic stability after 8000 cycles tested at $1.6 \mathrm{~A} \mathrm{~g}^{-1}$ (Fig. 9).

This is amongst the highest energy and power density reported so far for a hybrid system. One of the explanations to this increased capacitance is the faradic reaction of nitrogenous group combined with the improved wettability of the pores walls. The other explanation is that doping carbon material with nitrogen or other heteroatoms improves the conductivity of the carbon materials which in turn results in better electrochemical performance.

The exceptional outcome of the above reported nitrogen doped activated carbon can be considered as a potential cathode material for a LiHSC.

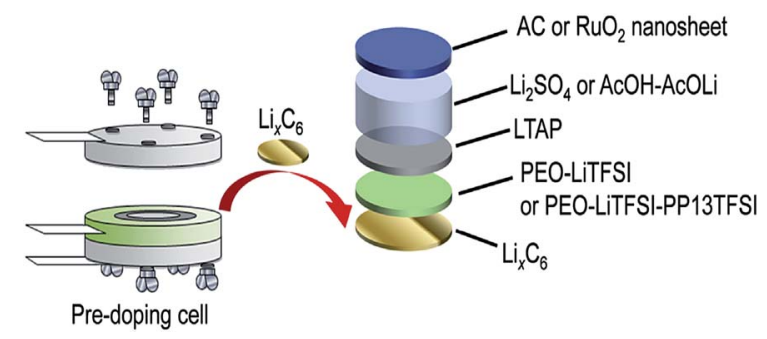

Fig. 8 Schematic illustration of the design of $\mathrm{Li}^{+}$pre-doped aqueous $\mathrm{LiC}$ with multi-layered $\mathrm{Li}_{x} \mathrm{C}_{6}$ negative electrode. Reproduced with permission. ${ }^{123}$ ○) 2016 Elsevier.

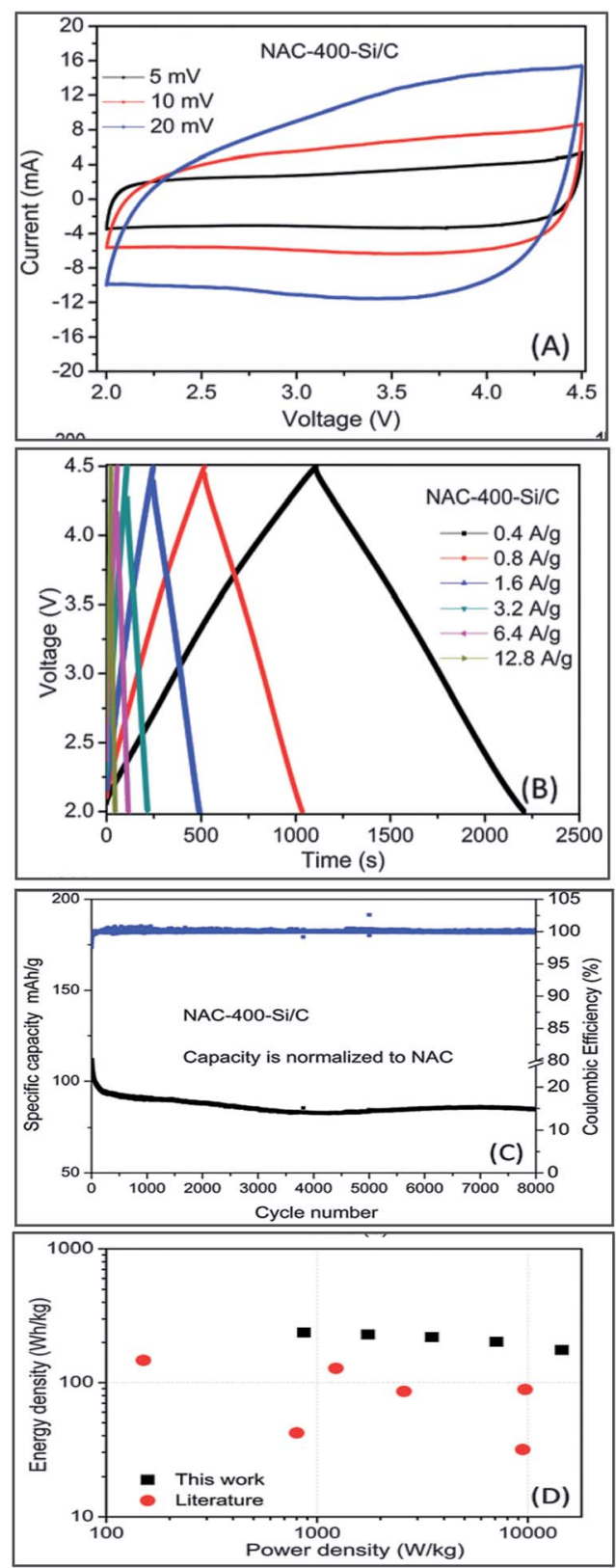

Fig. 9 (a) CV diagrams of the NAC-400-Si/C HSC at various scan rates (b) CD of the NAC-400-Si/C hybrid cell (c) cycling performance of the NAC-400-Si/C HSC at $0.4 \mathrm{~A} \mathrm{~g}^{-1}$ (d) Ragone plot of the NAC-400-Si/C HSC. Reproduced with permission. ${ }^{36}$ (C) 2015 The Royal Society of Chemistry.

\section{Conclusion \& future perspectives}

In summary, Li-HSC is indeed a promising energy storage system because of their high electrochemical performance and long cyclic life. In comparison with all other energy storage devices including LIBs, SCs, fuel cells etc., LIHSCs are the only energy storage system which offers both a high energy density and a high power density so they have potential to meet the requirement of the smart energy storage systems. Graphene and activated carbons offer high thermal and physical stability, 
tuneable porous structure, high surface area and hence they have great use in the LIHSC. Nitrogen doping is of a great interest because of the high energy density govern by electrodes based on the nitrogen doped graphene and activated carbons. The more number of nitrogen dopant groups, the more is the energy density of electrodes. This high energy density is due to the faradic reaction of nitrogenous groups and the improved wettability of pore walls. Nitrogen doping also increases the conductivity of carbon atoms which in turns improves the capacity of the electrodes. However, there are still some problems which need to be solved before the commercialization of LiHSCs:

1. Main limitation of LIHSCs is the disproportionate power capability of the sluggish Faradaic lithium-intercalation anode and rapid non-Faradaic capacitive cathode. To tackle this, the chemistry between the electrodes is an essential key for understanding the potential of electrodes to balance the performance in LIHSC.

2. Graphene and activated carbon electrodes doped with nitrogen have better performance among all other electrodes because of additional charge storage mechanism induced by nitrogen. However, the effect of percentage doping is yet to be explained.

3. The other problem is about replacing commercial graphite by graphene or activated carbon which can only be successful by attaining the full electrochemical performance of graphene and activated carbon with/without the fabrication of composites.

4. We should keep in mind that to design and commercialize the LIHSC, electrodes interaction with each other is not the only issue but their reaction with electrolyte, current collectors, separators, and the electrodes preparation method is of great importance also. If these issues can be fully understood, only then we can hope for a breakthrough in energy industry.

\section{Acknowledgements}

The authors gratefully acknowledge the financial support from Ministry of Science and Technology of China (No. 2016YFA0202900), the National Natural Science Foundation of China (NSFC, 51072117, 51672173), Guangdong Province (2016A010103018).

\section{References}

1 F. Schipper and D. Aurbach, Russ. J. Electrochem., 2016, 52, 1095-1121.

2 J. Yan, Q. Wang, T. Wei and Z. Fan, Adv. Energy Mater., 2014, 4, 1300816.

3 J. B. Goodenough and K.-S. Park, J. Am. Chem. Soc., 2013, 135, 1167-1176.

4 E. M. Erickson, C. Ghanty and D. Aurbach, J. Phys. Chem. Lett., 2014, 5, 3313-3324.

5 P. Rozier and J. M. Tarascon, J. Electrochem. Soc., 2015, 162, A2490-A2499.

6 B. Scrosati and J. Garche, J. Power Sources, 2010, 195, 24192430.
7 Y. Li, C. Zhu, T. Lu, Z. Guo, D. Zhang, J. Ma and S. Zhu, Carbon, 2013, 52, 565-573.

8 J.-M. Tarascon and M. Armand, Nature, 2001, 414, 359-367.

9 V. Etacheri, R. Marom, R. Elazari, G. Salitra and D. Aurbach, Energy Environ. Sci., 2011, 4, 3243-3262.

10 A. Manthiram, J. Phys. Chem. Lett., 2011, 2, 176-184.

11 Z. Niu, L. Liu, L. Zhang, W. Zhou, X. Chen and S. Xie, Adv. Energy Mater., 2015, 5, 1500677.

12 L. L. Zhang and X. Zhao, Chem. Soc. Rev., 2009, 38, 2520-2531.

13 W. Zhang, H. Lin, Z. Lin, J. Yin, H. Lu, D. Liu and M. Zhao, ChemSusChem, 2015, 8, 2114-2122.

14 Y. Dong, H. Lin, D. Zhou, H. Niu, Q. Jin and F. Qu, Electrochim. Acta, 2015, 159, 116-123.

15 L. Li, Z. Wu, S. Yuan and X.-B. Zhang, Energy Environ. Sci., 2014, 7, 2101-2122.

16 M. Winter and R. J. Brodd, Chem. Rev., 2005, 105, 1021.

17 S. Hashmi, Natl. Acad. Sci. Lett., 2004, 27, 27-46.

18 A. González, E. Goikolea, J. A. Barrena and R. Mysyk, Renewable Sustainable Energy Rev., 2016, 58, 1189-1206.

19 A. J. Amali, J.-K. Sun and Q. Xu, Chem. Commun., 2014, 50, 1519-1522.

20 Y. Deng, Y. Xie, K. Zou and X. Ji, J. Mater. Chem. A, 2016, 4, 1144-1173.

21 Y. Zhang, H. Feng, X. Wu, L. Wang, A. Zhang, T. Xia, H. Dong, X. Li and L. Zhang, Int. J. Hydrogen Energy, 2009, 34, 4889-4899.

22 P. Simon and Y. Gogotsi, Nat. Mater., 2008, 7, 845-854.

23 L. Staaf, P. Lundgren and P. Enoksson, Nano Energy, 2014, 9, 128-141.

24 F. Ran, X. Zhang, Y. Liu, K. Shen, X. Niu, Y. Tan, L. Kong, L. Kang, C. Xu and S. Chen, RSC Adv., 2015, 5, 87077-87083.

25 Y. Shao, M. F. El-Kady, L. J. Wang, Q. Zhang, Y. Li, H. Wang, M. F. Mousavi and R. B. Kaner, Chem. Soc. Rev., 2015, 44, 3639-3665.

26 B. G. S. Raj, R. N. R. Ramprasad, A. M. Asiri, J. J. Wu and S. Anandan, Electrochim. Acta, 2015, 156, 127-137.

27 Y. Zhai, Y. Dou, D. Zhao, P. F. Fulvio, R. T. Mayes and S. Dai, Adv. Mater., 2011, 23, 4828-4850.

28 Y. Gogotsi and P. Simon, Science, 2011, 334, 917-918.

29 A. S. Aricò, P. Bruce, B. Scrosati, J.-M. Tarascon and W. Van Schalkwijk, Nat. Mater., 2005, 4, 366-377.

30 D. P. Dubal, O. Ayyad, V. Ruiz and P. Gomez-Romero, Chem. Soc. Rev., 2015, 44, 1777-1790.

31 X. Lu, M. Yu, G. Wang, Y. Tong and Y. Li, Energy Environ. Sci., 2014, 7, 2160-2181.

32 K. Naoi, S. Ishimoto, J.-i. Miyamoto and W. Naoi, Energy Environ. Sci., 2012, 5, 9363-9373.

33 V. Aravindan, J. Gnanaraj, Y.-S. Lee and S. Madhavi, Chem. Rev., 2014, 114, 11619-11635.

34 Z. Caban-Huertas, D. P. Dubal and P. Gómez-Romero, ECS Meeting Abstracts, 2016, no. 2, p. 867.

35 Y. Ma, H. Chang, M. Zhang and Y. Chen, Adv. Mater., 2015, 27, 5296-5308.

36 B. Li, F. Dai, Q. Xiao, L. Yang, J. Shen, C. Zhang and M. Cai, Energy Environ. Sci., 2016, 9, 102-106.

37 M. Sevilla and R. Mokaya, Energy Environ. Sci., 2014, 7, 1250-1280. 
38 D. Cericola and R. Kötz, Electrochim. Acta, 2012, 72, 1-17. 39 H. Ma, J. He, D.-B. Xiong, J. Wu, Q. Li, V. Dravid and Y. Zhao, ACS Appl. Mater. Interfaces, 2016, 8, 1992-2000.

40 K. Leng, F. Zhang, L. Zhang, T. Zhang, Y. Wu, Y. Lu, Y. Huang and Y. Chen, Nano Res., 2013, 6, 581-592.

41 X. Sun, X. Zhang, H. Zhang, N. Xu, K. Wang and Y. Ma, J. Power Sources, 2014, 270, 318-325.

42 H. Konno, T. Kasashima and K. Azumi, J. Power Sources, 2009, 191, 623-627.

43 V. Aravindan, N. Shubha, W. C. Ling and S. Madhavi, J. Mater. Chem. A, 2013, 1, 6145-6151.

44 J. H. Lee, W. H. Shin, S. Y. Lim, B. G. Kim and J. W. Choi, Materials for Renewable and Sustainable Energy, 2014, 3, 22.

45 W. Ahn, D. U. Lee, G. Li, K. Feng, X. Wang, A. Yu, G. Lui and Z. Chen, ACS Appl. Mater. Interfaces, 2016, 8, 25297-25305.

46 K. Naoi, K. Kisu, E. Iwama, S. Nakashima, Y. Sakai, Y. Orikasa, P. Leone, N. Dupré, T. Brousse and P. Rozier, Energy Environ. Sci., 2016, 9, 2143-2151.

47 M. Zhi, C. Xiang, J. Li, M. Li and N. Wu, Nanoscale, 2013, 5, 72-88.

48 W.-H. Qu, F. Han, A.-H. Lu, C. Xing, M. Qiao and W.-C. Li, J. Mater. Chem. A, 2014, 2, 6549-6557.

49 Y. Lei, Z.-H. Huang, Y. Yang, W. Shen, Y. Zheng, H. Sun and F. Kang, Sci. Rep., 2013, 3, 2477.

50 K. Naoi, S. Ishimoto, J.-i. Miyamoto and W. Naoi, Energy Environ. Sci., 2012, 5, 9363-9373.

51 M. D. Stoller and R. S. Ruoff, Energy Environ. Sci., 2010, 3, 1294-1301.

$52 \mathrm{H}$. Khasawneh and M. Illindala, IEEE Trans. Ind. Appl., 2015, 51, 1962-1969.

53 V. V. N. Obreja, Phys. E, 2008, 40, 2596-2605.

54 A. Cultura II and Z. Salameh, cell, 2015, 1, 1.

55 M. He, K. Fic, E. Fra, P. Novák and E. J. Berg, Energy Environ. Sci., 2016, 9, 623-633.

56 S. Zhang and N. Pan, Adv. Energy Mater., 2015, 5, 1401401.

57 N. Goubard-Bretesché, O. Crosnier, F. Favier and T. Brousse, Electrochim. Acta, 2016, 206, 458-463.

58 A. Balducci, U. Bardi, S. Caporali, M. Mastragostino and F. Soavi, Electrochem. Commun., 2004, 6, 566-570.

59 H. Wang, M. Yoshio, A. K. Thapa and H. Nakamura, J. Power Sources, 2007, 169, 375-380.

60 H. Wang, Y. Liang, T. Mirfakhrai, Z. Chen, H. S. Casalongue and H. Dai, Nano Res., 2011, 4, 729-736.

61 X. Cai, S. H. Lim, C. K. Poh, L. Lai, J. Lin and Z. Shen, J. Power Sources, 2015, 275, 298-304.

62 W. Liu, X. Li, M. Zhu and X. He, J. Power Sources, 2015, 282, 179-186.

63 F. Sun, J. Gao, X. Liu, L. Wang, Y. Yang, X. Pi, S. Wu and Y. Qin, Electrochim. Acta, 2016, 213, 626-632.

64 T. Kim, G. Jung, S. Yoo, K. S. Suh and R. S. Ruoff, ACS Nano, 2013, 7, 6899-6905.

65 Y. Li, Y. Zhao, H. Cheng, Y. Hu, G. Shi, L. Dai and L. Qu, J. Am. Chem. Soc., 2011, 134, 15-18.

66 J. Lee, D. Wong, J. Velasco Jr, J. Rodriguez-Nieva, S. Kahn, P. Vo, H.-Z. Tsai, T. Taniguchi, K. Watanabe, A. Zettl, F. Wang, L. Levitov and M. Crommie, 2016, http:// meetings.aps.org/Meeting/MAR16/Event/261882.
67 P. Ruffieux, S. Wang, B. Yang, C. Sánchez-Sánchez, J. Liu, T. Dienel, L. Talirz, P. Shinde, C. A. Pignedoli and D. Passerone, Nature, 2016, 531, 489-492.

68 Y. Zhao, X. Li, B. Yan, D. Li, S. Lawes and X. Sun, J. Power Sources, 2015, 274, 869-884.

69 S. Chen, J. Duan, M. Jaroniec and S. Z. Qiao, J. Mater. Chem. A, 2013, 1, 9409-9413.

70 Y. Li, Z. Li and P. K. Shen, Adv. Mater., 2013, 25, 2474-2480.

71 A. Amiri, M. Shanbedi, G. Ahmadi, H. Eshghi, S. N. Kazi, B. T. Chew, M. Savari and M. N. M. Zubir, Sci. Rep., 2016, 6, 32686.

72 C. Zhu, T. Y.-J. Han, E. B. Duoss, A. M. Golobic, J. D. Kuntz, C. M. Spadaccini and M. A. Worsley, Nat. Commun., 2015, 6, 6962.

73 X. Wu, Z. Xie, M. Sun, T. Lei, Z. Zuo, X. Xie, Y. Liang and Q. Huang, RSC Adv., 2016, 6, 90384-90387.

74 Z. Wen, X. Wang, S. Mao, Z. Bo, H. Kim, S. Cui, G. Lu, X. Feng and J. Chen, Adv. Mater., 2012, 24, 5610-5616.

75 D.-W. Wang, F. Li, Z.-G. Chen, G. Q. Lu and H.-M. Cheng, Chem. Mater., 2008, 20, 7195-7200.

76 J. P. Paraknowitsch and A. Thomas, Energy Environ. Sci., 2013, 6, 2839-2855.

77 H. Wang, T. Maiyalagan and X. Wang, ACS Catal., 2012, 2, 781-794.

78 D. Li, L. Zhang, H. Chen, J. Wang, L.-X. Ding, S. Wang, P. J. Ashman and H. Wang, J. Mater. Chem. A, 2016, 4, 8630-8635.

79 Y.-P. Lin, Y. Ksari, D. Aubel, S. Hajjar-Garreau, G. Borvon, Y. Spiegel, L. Roux, L. Simon and J.-M. Themlin, Carbon, 2016, 100, 337-344.

80 Z. Sun, Z. Yan, J. Yao, E. Beitler, Y. Zhu and J. M. Tour, Nature, 2010, 468, 549-552.

81 H. Huang, G. Luo, L. Xu, C. Lei, Y. Tang, S. Tang and Y. Du, Nanoscale, 2015, 7, 2060-2068.

82 J. Wu, L. Ma, R. M. Yadav, Y. Yang, X. Zhang, R. Vajtai, J. Lou and P. M. Ajayan, ACS Appl. Mater. Interfaces, 2015, 7, 14763-14769.

83 J. Li, Y. Zhang, X. Zhang, J. Han, Y. Wang, L. Gu, Z. Zhang, X. Wang, J. Jian and P. Xu, ACS Appl. Mater. Interfaces, 2015, 7, 19626-19634.

84 Y. Liao, Y. Gao, S. Zhu, J. Zheng, Z. Chen, C. Yin, X. Lou and D. Zhang, ACS Appl. Mater. Interfaces, 2015, 7, 19619-19625.

85 G. Luo, L. Liu, J. Zhang, G. Li, B. Wang and J. Zhao, ACS Appl. Mater. Interfaces, 2013, 5, 11184-11193.

86 C. Ma, X. Shao and D. Cao, J. Mater. Chem., 2012, 22, 89118915.

87 Y. Xu, Y. Mo, J. Tian, P. Wang, H. Yu and J. Yu, Appl. Catal., $B, 2016,181,810-817$.

88 A. L. M. Reddy, A. Srivastava, S. R. Gowda, H. Gullapalli, M. Dubey and P. M. Ajayan, ACS Nano, 2010, 4, 6337-6342.

89 H. M. Jeong, J. W. Lee, W. H. Shin, Y. J. Choi, H. J. Shin, J. K. Kang and J. W. Choi, Nano Lett., 2011, 11, 2472-2477.

90 T. Brousse, D. Bélanger and J. W. Long, J. Electrochem. Soc., 2015, 162, A5185-A5189.

91 Y.-H. Lee, K.-H. Chang and C.-C. Hu, J. Power Sources, 2013, 227, 300-308. 
92 G. Hasegawa, T. Deguchi, K. Kanamori, Y. Kobayashi, H. Kageyama, T. Abe and K. Nakanishi, Chem. Mater., 2015, 27, 4703-4712.

93 Y. Deng, Y. Xie, K. Zou and X. Ji, J. Mater. Chem. A, 2016, 4, 1144-1173.

94 R. Raccichini, A. Varzi, S. Passerini and B. Scrosati, Nat. Mater., 2015, 14, 271-279.

95 M. Srivastava, J. Singh, T. Kuila, R. K. Layek, N. H. Kim and J. H. Lee, Nanoscale, 2015, 7, 4820-4868.

96 J. Zhu, D. Yang, Z. Yin, Q. Yan and H. Zhang, Small, 2014, 10, 3480-3498.

97 M. D. Stoller, S. Park, Y. Zhu, J. An and R. S. Ruoff, Nano Lett., 2008, 8, 3498-3502.

98 L. Tang, Y. Wang, Y. Li, H. Feng, J. Lu and J. Li, Adv. Funct. Mater., 2009, 19, 2782-2789.

99 B. Xu, S. Yue, Z. Sui, X. Zhang, S. Hou, G. Cao and Y. Yang, Energy Environ. Sci., 2011, 4, 2826-2830.

100 K. Fu, Y. Wang, C. Yan, Y. Yao, Y. Chen, J. Dai, S. Lacey, Y. Wang, J. Wan, T. Li, Z. Wang, Y. Xu and L. Hu, Adv. Mater., 2016, 28, 2587-2594.

101 H.-Q. Wang, G.-H. Yang, L.-S. Cui, Z.-S. Li, Z.-X. Yan, X.-H. Zhang, Y.-G. Huang and Q.-Y. Li, J. Mater. Chem. A, 2015, 3, 21298-21307.

102 Y. Shi, L. Wen, G. Zhou, J. Chen, S. Pei, K. Huang, H.-M. Cheng and F. Li, 2D Mater., 2015, 2, 024004.

103 F. Xiang, R. Mukherjee, J. Zhong, Y. Xia, N. Gu, Z. Yang and N. Koratkar, Energy Storage Materials, 2015, 1, 9-16.

104 D. Mhamane, V. Aravindan, M.-S. Kim, H.-K. Kim, K. C. Roh, D. Ruan, S. H. Lee, M. Srinivasan and K.-B. Kim, J. Mater. Chem. A, 2016, 4, 5578-5591.

105 H. Wang, C. Guan, X. Wang and H. J. Fan, Small, 2015, 11, 1470-1477.

106 Y. Ma, H. Chang, M. Zhang and Y. Chen, Adv. Mater., 2015, 27, 5296-5308.

107 L. Ye, Q. Liang, Y. Lei, X. Yu, C. Han, W. Shen, Z.-H. Huang, F. Kang and Q.-H. Yang, J. Power Sources, 2015, 282, 174178.
108 T. Zhang, F. Zhang, L. Zhang, Y. Lu, Y. Zhang, X. Yang, Y. Ma and Y. Huang, Carbon, 2015, 92, 106-118.

109 R. Xue, J. Yan, L. Jiang and B. Yi, Mater. Chem. Phys., 2015, 160, 375-382.

110 L. Zhang, K. Hui, K. Hui, X. Chen, R. Chen and H. Lee, Int. J. Hydrogen Energy, 2016, 41, 9443-9453.

111 J. H. Lee, W. H. Shin, M. H. Ryou, J. K. Jin, J. Kim and J. W. Choi, ChemSusChem, 2012, 5, 2328-2333.

112 R. Aswathy, T. Kesavan, K. Kumaran and P. Ragupathy, J. Mater. Chem. A, 2015, 3, 12386-12395.

113 Y. Liu, D. Zhang, Y. Shang, Y. Liu and J. Zhang, J. Electrochem. Soc., 2015, 162, A2123-A2130.

114 A. Pandolfo and A. Hollenkamp, J. Power Sources, 2006, 157, 11-27.

115 K. Karthikeyan, S. Amaresh, S. N. Lee, X. Sun, V. Aravindan, Y. G. Lee and Y. S. Lee, ChemSusChem, 2014, 7, 1435-1442.

116 L. Wei and G. Yushin, Nano Energy, 2012, 1, 552-565.

117 A. M. Abioye and F. N. Ani, Renewable Sustainable Energy Rev., 2015, 52, 1282-1293.

118 H. Feng, H. Hu, H. Dong, Y. Xiao, Y. Cai, B. Lei, Y. Liu and M. Zheng, J. Power Sources, 2016, 302, 164-173.

119 C. H. Kim, J.-H. Wee, Y. A. Kim, K. S. Yang and C.-M. Yang, J. Mater. Chem. A, 2016, 4, 4763-4770.

120 B. Bakhmatyuk, Electrochim. Acta, 2015, 163, 167-173.

121 B. Babu, P. Lashmi and M. Shaijumon, Electrochim. Acta, 2016, 211, 289-296.

122 S. Senthilkumar, N. Fu, Y. Liu, Y. Wang, L. Zhou and H. Huang, Electrochim. Acta, 2016, 211, 411-419.

123 S. Makino, R. Yamamoto, S. Sugimoto and W. Sugimoto, J. Power Sources, 2016, 326, 711-716.

124 E. Lim, C. Jo, H. Kim, M.-H. Kim, Y. Mun, J. Chun, Y. Ye, J. Hwang, K.-S. Ha and K. C. Roh, ACS Nano, 2015, 9, 7497-7505.

125 L. Zhang, X. Yang, F. Zhang, G. Long, T. Zhang, K. Leng, Y. Zhang, Y. Huang, Y. Ma and M. Zhang, J. Am. Chem. Soc., 2013, 135, 5921-5929. 